\title{
Predictors of Pharmacy Students' Intentions to Monitor Diabetes
}

\author{
Lisa M Guirguis ${ }^{a}$, Betty A Chewning ${ }^{b}$, Mara A Kieser \\ ${ }^{a}$ Faculty of Pharmacy and Pharmaceutical Sciences, University of Alberta, Edmonton, AB, Canada \\ ${ }^{\mathrm{b}} \mathrm{School}$ of Pharmacy, University of Wisconsin, Madison, WI, USA.
}

Received November 10, 2008; Revised March 23, 2009; Accepted March 25, 2009; Published, March $28,2009$.

\begin{abstract}
Purpose. This research explores predictors of pharmacy students' adoption of one specific behavior, monitoring diabetes ABCs (A1c, blood pressure, and cholesterol) in the community pharmacy. Specifically, this research assessed which student situation and attitudinal factors are predictors of students' intentions and behavior in asking patients about the diabetes targets and goals as per a conceptual model. Methods. Data was drawn from a randomized controlled trial to assess the impact of the diabetes check in pharmacy students during their community pharmacy clerkships. A survey measured students' self-efficacy, outcome expectancies, role beliefs, mattering as well as students' experiences with the Diabetes Check and intentions to routinely monitor diabetes. Stepwise hierarchical multiple linear regression reflected the conceptual model and was used to assess the research questions. Results. Survey response rate was $94 \%$ and analysis was performed on a sample of 118 students. In summary, pharmacy students' intentions and monitoring behaviors were predicted by the students' situation and attitudes. Specifically, students' intentions to ask patients about the diabetes ABCs were predicted by pharmacy site counseling, monitoring role beliefs, self-efficacy, and positive outcome expectancies. Mattering predicted intentions, but differently in each study group. Behavior in asking about patients with diabetes about blood pressure and cholesterol was predicted by pharmacy site counseling, self efficacy, and monitoring role beliefs. Students' behavior in asking about A1c was pharmacy site counseling, self efficacy, and monitoring role beliefs in additional to completing the Diabetes Check assignment. Conclusions. Monitoring intentions and behaviors were consistently predicted by pharmacy site counseling, monitoring role beliefs, and self-efficacy and future research investigating the pharmacists' behavior should include these variables. The role of mattering and outcome expectancies in predicting monitoring intentions requires further study.
\end{abstract}

\section{INTRODUCTION}

Pharmacists and have been mandated to change their practice behaviors from a product focused to a patient focused practice. Students are frequently called on to carry the pharmacy profession forward and provide patient-centered care (1). While progress has been made and many practitioners have established patient focused practices, the bulk of pharmacists focus their practice on providing a product to a patient. It is important to understand what factors pharmacists influence change to patient-focused care.

We propose studying predictors of pharmacy students' adoption of one specific behavior, assessing patients' diabetes target levels and goals. Pharmacy students were trained to ask patients with diabetes about their A1c, blood pressure, and cholesterol (diabetes ABCs) as part of a clerkship requirement, titled the Diabetes Check. This training involved providing tools and resources to students, viewing model, and rehearsal in both a controlled environment and the pharmacy site (2). The intervention included a tool from the American Diabetes Association which explained the diabetes ABCs partnered with an interview guide which modeled the structure of the patient interaction (3). Subsequently, we assessed pharmacy students' application of these patient-focused techniques to their patient interactions at a clerkship site.

\footnotetext{
Corresponding Author: Lisa Guirguis, Assistant Professor, Faculty of Pharmacy and Pharmaceutical Sciences, 3126 Dentistry/Pharmacy Centre, University of Alberta, Edmonton, Alberta CANADA, Email: lguirguis@pharmacy.ualberta.ca
} 
The Diabetes Check was incorporated into the required ambulatory pharmaceutical care clerkship block as part of their final year of their entry level PharmD. In the ambulatory pharmaceutical care clerkship, students were assigned to six to eight weeks in one of 39 community pharmacies. This unique training model allows students to perform patient centered monitoring activities in all community pharmacy clerkship sites.

We choose to focus this research on diabetes, pharmacy students, and behavioral models for the following reasons. Diabetes has been considered an epidemic. More than 180 million people worldwide have diabetes and this number is likely to more than double by 2030 (4). In addition, diabetes causes about $5 \%$ of all deaths globally each year (4). Diabetes tools have been developed and promoted to help health care professionals have brief conversations about assessing the diabetes ABCs in patients with diabetes.

Pharmacy students have the prerequisite knowledge to assess diabetes. More specifically, students are taught the importance of monitoring diabetes goals and numbers in their coursework. However, the training students receive at school is not routinely transferred to community practice. The majority of time in clerkships is spent on dispensing medications, not patient care activities $(5,6)$. Thus, students are not routinely providing care such as the Diabetes Check allowing for us to assess a change in behavior.

Three theories informed the conceptual model (Figure 1) used to predict pharmacy students' intentions and behaviors: Social Cognitive Theory, Role Theory, and Mattering. First, social cognitive theory suggests that students who have the skills and self-efficacy to ask patients about their diabetes goals and expect positive outcomes would be more likely to perform a Diabetes Check in the future (7). Previously, self-efficacy scales have assessed pharmacy students' self efficacy in clerkship activities $(8,9)$. Second, role theory suggests that students with more positive role beliefs toward monitoring after experience with the Diabetes Check would have greater intentions to monitor patients in the future. In the pharmacy practice literature, pharmacists' role orientations or beliefs have been shown to influence patientpharmacist interactions (10). Finally, mattering posits that students who feel they make a difference to patients may be more likely to conduct a Diabetes Check $(11,12)$.

In summary, this research explores the dynamics underlying students' intentions and behaviors in regards to asking about the diabetes ABCs as per a conceptual model. This novel analysis assesses the influence of both the pharmacy site and student attitudes using recognized theory on students' monitoring behaviors in "real-life" community pharmacies. Currently, it is not known what predicts students' use of patient-focused techniques in their clerkship training.

\section{METHODS}

This study used a stepwise hierarchical multiple linear regression based on data was drawn from a randomized controlled trial to assess the impact of the diabetes check (2). The study sample consisted of the entire population of fourth year professional pharmacy students (approximately 130) at the University of Wisconsin-Madison School of Pharmacy between May 2005 and May 2006.

Both groups completed an assignment on how to monitor diabetes in a community pharmacy and a brief training session was provided separately to study groups. The primary difference was that intervention group students were required to interact with 5 to 10 patients and focus on monitoring the diabetes ABCs whereas students in the control group were not required to interact with patients and were encouraged to monitor all aspects of diabetes for only 2 patients. An in-depth review would take more time than monitoring the diabetes ABCs; thus pharmacy students in the control group were only required to complete 2 reviews. Results of the randomized controlled trial found the Diabetes Check improved pharmacy students' monitoring behavior for A1c and general counseling beliefs (2).

\section{Measures}

Students completed a survey after completing their assignment using Survey Monkey ${ }^{\circledR}$ (www.surveymonkey.com), an online survey tool. Students could only submit one required survey using this technology. 
Figure 1 Conceptual Model of Pharmacy Student Behaviors

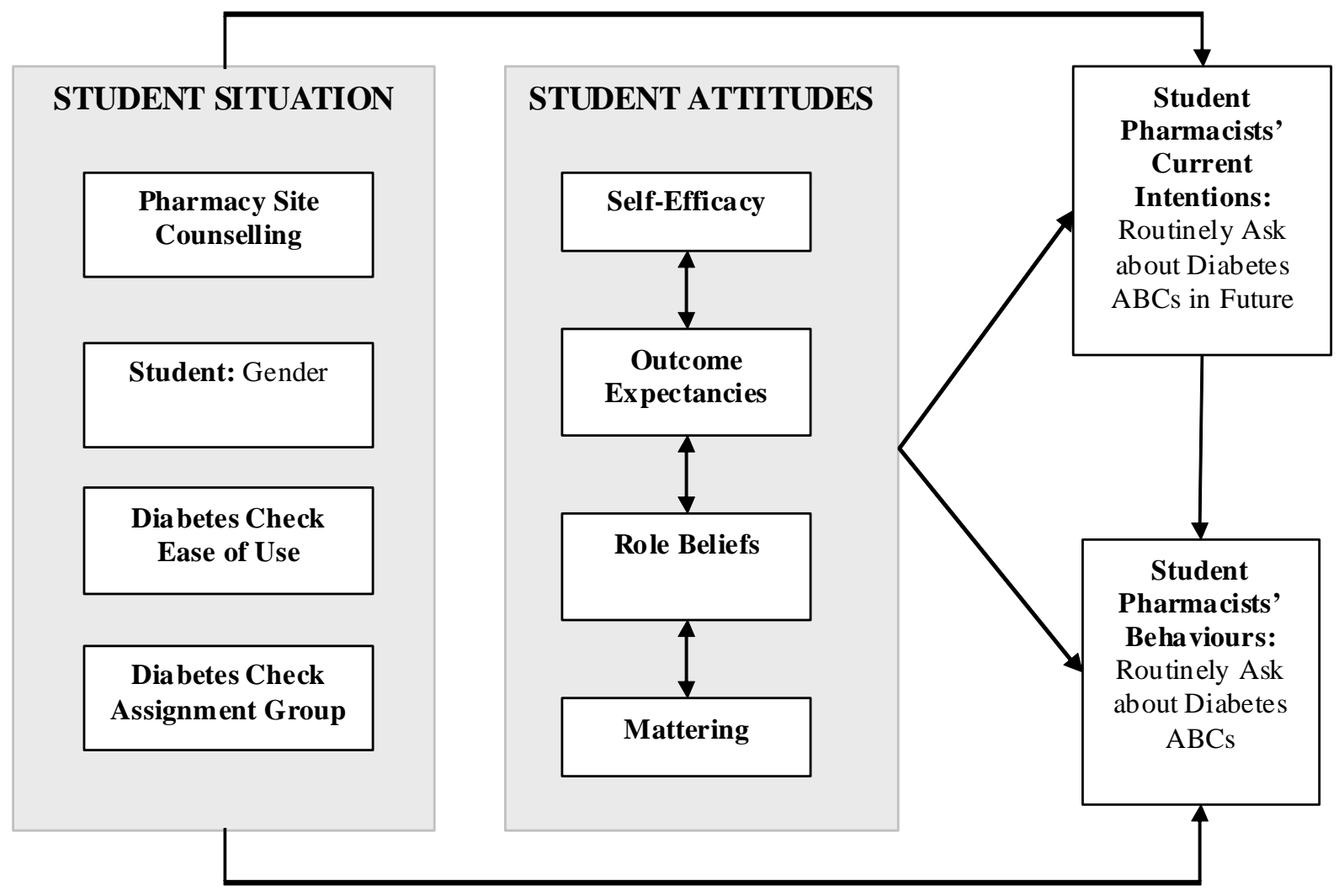

Behavior was assessed by three questions which asked how often students asked patients with diabetes about their A1c, blood pressure, or cholesterol numbers with a seven item response scale. See Table 1 for the response options for all questions. When possible a seven item response scale was used to increase variance; however fewer items were used with validated scales or to facilitate understanding (13). The survey assessed students' self-efficacy, outcome expectancies, role beliefs, and mattering as well as students' experiences with the Diabetes Check and intentions to routinely ask about diabetes ABCs. The complete survey and measure development with reliability and validity are described elsewhere (13). The self-efficacy scale assessed how sure pharmacy students were that they could ask patients about the diabetes ABC in various conditions. A sample questions reads, "The following set of questions refers to your interactions with patients with diabetes. How sure you that you could are: routinely ask patients at medication refills about A1c, blood pressure, and cholesterol?" The outcome expectancies scale assessed the frequency of the expected outcome of asking patient about the diabetes ABCs and includes the following question, "If you ask patients with diabetes routinely about their A1c, blood pressure and cholesterol how often do you think this practice will open up opportunities to talk with patients?” (7).

The role belief scales measure the strength of the beliefs that pharmacy student have about their role counseling patients as measured by the counseling role orientation scale (CRO) or more specifically asking about the Diabetes ABCs with the Monitoring Role Orientation (MRO) scale. A sample CRO question is "Most patients are more interested in receiving quick and inexpensive service from their pharmacist rather than advice." A sample MRO question reads, "Community pharmacists should talk to patients with diabetes about A1c, blood pressure, and cholesterol at every prescription refill."

The mattering scale assessed the degree to which students felt that they were acknowledged and relevant to patients and includes questions such 
as "To what extent do you "make a difference" to patients?" The pharmacy site counseling variable was a composed of two questions on patients' expectations for counseling (How many patient expect pharmacists to counsel with every prescription?) and preceptor's frequency of asking about the diabetes ABCs (How many patients with diabetes does your clinical instructor ask about the diabetes ABC?). Both questions had seven response items: (1=no patients. 2=almost no patients, $3=$ less than half, $4=$ half of patients, $5=$ more than half, $6=$ almost all, $7=$ all patients). This research was reviewed by the UW-Madison Health Sciences Institutional Review Board.

We hypothesized that the intervention, student and pharmacy site characteristics would influence student change in attitudes which would in turn influence intentions to ask about the Diabetes ABCs in the future and ultimately students' behavior asking patients about the Diabetes ABCs.

\section{ANALYSIS}

Stepwise hierarchical multiple linear regression was used to assess the research questions. Research question one was tested with two blocks of variables. Order of entry reflected the conceptual model; ease of use, pharmacy site counseling, group, and gender were entered in variable block one, and attitude variables (i.e., sense of self-efficacy, outcome expectations, role beliefs, and mattering) in variable block two. Research question two was tested with three blocks of variables. Variable blocks one and two were entered as per research questions one, and the third variable block had intentions as per the conceptual model.

As the most parsimonious model is best, a correlation matrix consisting of the independent variables was examined and factors with correlations greater than 0.8 were eliminated from the analysis. Regression assumptions were examined and outliers with significant influence on the model were removed.

\section{RESULTS}

\section{Sample}

Of 130 students in the clerkship, three students dropped out for a total of 127 students. Survey response rate was $94 \%$ (119 of 127 students). The sample was $71 \%$ female. Table 1 describes the scale responses for the behaviours, intentions, and student situation.

Data were also examined for outliers. In preliminary analysis, one student had a consistent impact on the data, as assessed by regression residuals, leverage, and Cook's D statistic. This student was also marked as an outlier on scatterplots and histograms. This student attitude scores indicated positive attitudes about pharmacists' counseling in general, but not about asking about the diabetes ABCs. This student wrote an exceptionally negative comment when asked to describe the assignment. It read, "Not necessary the doctor should take care of this; unless I am a working in a clinic with the doctor". Weighing all these factors a decision was made to eliminate this students' data was eliminated from all analysis leaving sample size of 118 .

\section{Reducing Variables in the Conceptual Model}

The first was to develop parsimonious models for predicting behavior and intentions. As the sample size was fixed at 118 students and the conceptual model contained nine independent variables, the independent variables were examined to determine if the number of variables in the conceptual model could be reduced. This was accomplished in three ways; (1) through the use of theory in the conceptual model, (2) by examination of a correlation matrix, and (3) by stepwise hierarchical linear regression.

The first step focused on the conceptual model itself. Debate exists on whether outcome expectancies have any independent contribution to the prediction of behavior when differences in selfefficacy are controlled (7, 14-16). Thus, asking about A1c, blood pressure, and cholesterol were separately regressed on self-efficacy and outcome expectancies was added in a second step. Over all three behaviors (i.e., A1c, blood pressure, and cholesterol separately), the change in $\mathrm{R}^{2}$ was not statistically significant $(\mathrm{p}>0.05)$, demonstrating that outcome expectancies did not explain any additional variance in behavior after controlling for self-efficacy. Thus, outcome expectancy scales were dropped from the models predicting behavior changes. No other theory was identified that could be used to reduce the model. 
Table 1 Descriptive of the Dependent and Independent Variables

\begin{tabular}{|c|c|c|c|c|c|c|}
\hline & $\mathrm{N}$ & Mean & Median & Std. Dev & Min & Max \\
\hline \multicolumn{7}{|l|}{ Behaviours } \\
\hline Ask About A1C & 116 & 3.84 & 4.00 & 1.28 & 1.00 & 6.00 \\
\hline Ask about Blood Pressure $^{a}$ & 117 & 4.26 & 4.00 & 1.26 & 2.00 & 7.00 \\
\hline Ask about Cholesterol $^{\mathrm{a}}$ & 117 & 3.91 & 4.00 & 1.28 & 1.00 & 7.00 \\
\hline \multicolumn{7}{|l|}{ Intention } \\
\hline Intention Scale $^{\mathrm{b}}$ & 117 & 4.55 & 4.75 & 0.75 & 3.00 & 6.00 \\
\hline \multicolumn{7}{|l|}{ Attitudes } \\
\hline Self-Efficacy Scale ${ }^{c}$ & 118 & 4.44 & 4.50 & 1.17 & 1.73 & 6.73 \\
\hline Positive Outcome Expectancies ${ }^{\mathrm{d}}$ & 117 & 5.48 & 5.50 & 0.85 & 3.00 & 7.00 \\
\hline Negative Outcome Expectancies ${ }^{\mathrm{d}}$ & 117 & 3.34 & 3.33 & 0.82 & 2.00 & 5.33 \\
\hline Counseling Role Orientation ${ }^{\mathrm{e}}$ & 117 & 5.67 & 5.71 & 0.67 & 3.43 & 6.86 \\
\hline Monitoring Role Orientation ${ }^{\mathrm{e}, \mathrm{f}}$ & 117 & 5.07 & 5.25 & 0.92 & 2.50 & 7.00 \\
\hline Mattering Scale ${ }^{\mathrm{g}}$ & 118 & 3.90 & 4.00 & 0.52 & 2.43 & 5.00 \\
\hline \multicolumn{7}{|l|}{ Student Situation } \\
\hline Ease of Use Diabetes Check ${ }^{\mathrm{h}}$ & 117 & 3.70 & 4.00 & 0.76 & 2.00 & 5.00 \\
\hline Pharmacy Site Counseling $^{\mathrm{a}}$ & 117 & 4.04 & 4.00 & 1.21 & 1.67 & 6.67 \\
\hline
\end{tabular}

Note: All responses were coded so higher numbers representing higher or more frequent outcomes.

a. Response Options: $1=$ no patients. $2=$ almost no patients, $3=$ less than half, $4=$ half of patients, $5=$ more than half, $6=$ almost all, $7=$ all patients.

b. Response Options: 1=Extremely unlikely, 2=Very unlikely, 3=unlikely, 4=Likely, 5=Very Likely, 6=Extremely likely.

c. Response Options: 1=Not sure at all, 2=Slightly sure, 3=Somewhat sure, 4=Rather sure, 5=Quite sure, 6=Very sure, 7=Extremely sure.

d. Response Options: $1=$ Never, $2=$ Almost never, $3=$ Less than half the time, $4=$ Half the time, $5=$ More than half the time, $6=$ Almost always, $7=$ Always.

e. Response Options: 1=Very strongly disagree, 2=Strongly disagree, 3=Disagree, 4=Neutral, 5=Agree, 6=Strongly agree,7=Very strongly agree.

f. Response Options: 1=Not important at all, 2=Slightly important, 3=Somewhat important, 4=Rather Important, 5=Quite Important, 6=Very important,

7=Extremely important.

g. Response Options: 1=Not at all, 2=Almost none at all, 3=A little, 4=Somewhat, 5=A great deal.

h. Response Options: 1=Extremely difficult, 2=Difficult, 3=Neutral, 4=Easy, 5=Extremely easy.

Next a correlation matrix of all behaviors, intentions, and attitudes was examined to assess the risk of serious multicollinearity and describe bivariate relationships between independent and dependent variables (Table 2). There were no correlations (Table 2) greater than 0.80 ; thus serious multicollinearity was not considered.

Next, a second correlation matrix was examined to determine if there were correlations between students who had completed the Diabetes Check Assignment and those who had not (Table 3). The Diabetes Check assignment has been designed to potentially influence students' attitudes; therefore we felt this interaction should be examined. An interaction between CRO score and group was considered for the analysis predicting intentions as CRO and intentions scores were positively correlated for the group who did not complete the Diabetes Check, but not correlated in the intervention group. Similarly in the group which completed the Diabetes Check, mattering scores were not correlated with all behaviors, intentions, and attitudes, while mattering scores were correlated with all behaviors, intentions and attitudes in the control group. The interaction between mattering and group was considered for all analyses.

\section{Intention Regression}

Using the conceptual model, hierarchical multiple linear regression was used to determine students' intentions to use a Diabetes Check in the future. Order of entry for the regression analysis reflected the conceptual model. Student situation variables including Diabetes Check assignment, gender, ease of use of the course assignment, and site counseling variable were entered in variable block one, and attitude variables were entered in variable block two along with a CRO and Diabetes Check assignment and a mattering and Diabetes Check assignment interaction term. 
Table 2 Correlation Matrix

\begin{tabular}{|c|c|c|c|c|c|c|c|c|c|c|}
\hline & & 1 & 2 & 3 & 4 & 5 & 6 & 7 & 8 & 9 \\
\hline Ask about A & 1 & $\begin{array}{l}1 \\
n=116\end{array}$ & & & & & & & & \\
\hline Ask about B & 2 & $\begin{array}{l}0.66^{*} \\
\mathrm{n}=116\end{array}$ & $\begin{array}{l}1 \\
n=117\end{array}$ & & & & & & & \\
\hline Ask about C & 3 & $\begin{array}{l}0.62^{*} \\
\mathrm{n}=116\end{array}$ & $0.77 *$ & $\begin{array}{l}1 \\
n=117\end{array}$ & & & & & & \\
\hline Intention & 4 & $\begin{array}{l}0.52^{*} \\
\mathrm{n}=115\end{array}$ & $\begin{array}{l}0.51^{*} \\
\mathrm{n}=116\end{array}$ & $\begin{array}{l}0.43^{*} \\
\mathrm{n}=116\end{array}$ & $\begin{array}{l}1 \\
n=117\end{array}$ & & & & & \\
\hline Self-Efficacy & 5 & $\begin{array}{l}0.57^{*} \\
\mathrm{n}=116\end{array}$ & $\begin{array}{l}0.61 * \\
n=117\end{array}$ & $\begin{array}{l}0.55^{*} \\
\mathrm{n}=117\end{array}$ & $\begin{array}{l}0.66^{*} \\
\mathrm{n}=117\end{array}$ & $\begin{array}{l}1 \\
n=118\end{array}$ & & & & \\
\hline Positive $\mathrm{OE}^{* *}$ & 6 & $\begin{array}{l}0.34^{*} \\
\mathrm{n}=115\end{array}$ & $\begin{array}{l}0.36^{*} \\
n=116\end{array}$ & $\begin{array}{l}0.23^{*} \\
\mathrm{n}=116\end{array}$ & $\begin{array}{l}0.56^{*} \\
\mathrm{n}=117\end{array}$ & $\begin{array}{l}0.47 * \\
\mathrm{n}=117\end{array}$ & $\begin{array}{l}1 \\
n=117\end{array}$ & & & \\
\hline Negative OE** & 7 & $\begin{array}{l}-0.40^{*} \\
\mathrm{n}=115\end{array}$ & $\begin{array}{l}-0.34 * \\
n=116\end{array}$ & $\begin{array}{l}-0.35^{*} \\
n=116\end{array}$ & $\begin{array}{l}-0.43^{*} \\
\mathrm{n}=117\end{array}$ & $\begin{array}{l}-0.51^{*} \\
\mathrm{n}=117\end{array}$ & $\begin{array}{l}-0.34^{*} \\
\mathrm{n}=117\end{array}$ & $\begin{array}{l}1 \\
n=117\end{array}$ & & \\
\hline CRO & 8 & $\begin{array}{l}\mathbf{0 . 1 2} \\
\mathrm{n}=115\end{array}$ & $\begin{array}{l}\mathbf{0 . 1 3} \\
n=116\end{array}$ & $\begin{array}{l}\mathbf{0 . 0 3} \\
\mathrm{n}=116\end{array}$ & $\begin{array}{l}0.36^{*} \\
n=117\end{array}$ & $\begin{array}{l}0.22 * \\
n=117\end{array}$ & $\begin{array}{l}0.27 * \\
\mathrm{n}=117\end{array}$ & $\begin{array}{l}-0.37 * \\
\mathrm{n}=117\end{array}$ & $\begin{array}{l}1 \\
n=117\end{array}$ & \\
\hline MRO & 9 & $\begin{array}{l}0.53^{*} \\
\mathrm{n}=115\end{array}$ & $\begin{array}{l}0.58^{*} \\
\mathrm{n}=116\end{array}$ & $\begin{array}{l}0.54^{*} \\
\mathrm{n}=116\end{array}$ & $\begin{array}{l}0.66^{*} \\
\mathrm{n}=117\end{array}$ & $\begin{array}{l}0.73^{*} \\
\mathrm{n}=117\end{array}$ & $\begin{array}{l}0.54^{*} \\
\mathrm{n}=117\end{array}$ & $\begin{array}{l}-0.50^{*} \\
\mathrm{n}=117\end{array}$ & $\begin{array}{l}0.29^{*} \\
\mathrm{n}=117\end{array}$ & $\begin{array}{l}1 \\
\mathrm{n}=117\end{array}$ \\
\hline Mattering & 10 & $\begin{array}{l}0.28 * \\
\mathrm{n}=116\end{array}$ & $\begin{array}{l}0.34 * \\
\mathrm{n}=117\end{array}$ & $\begin{array}{l}0.20 * \\
\mathrm{n}=117\end{array}$ & $\begin{array}{l}0.33^{*} \\
\mathrm{n}=117\end{array}$ & $\begin{array}{l}0.40^{*} \\
\mathrm{n}=118\end{array}$ & $\begin{array}{l}0.43^{*} \\
\mathrm{n}=117\end{array}$ & $\begin{array}{l}-0.31^{*} \\
\mathrm{n}=117\end{array}$ & $\begin{array}{l}0.42^{*} \\
\mathrm{n}=117\end{array}$ & $\begin{array}{l}0.32 * \\
\mathrm{n}=117\end{array}$ \\
\hline
\end{tabular}

(Non-significant correlations are bolded)

*Correlation is significant $(\mathrm{p}<0.05)$.

$* * \mathrm{OE}=$ Outcome Expectancies.

The regression model accounted for a significant amount of variance in intentions, with $\mathrm{F}(7,109)=23.77, \mathrm{p}<0.001, \mathrm{R}^{2}=0.60$ and adjusted $\mathrm{R}^{2}=0.58$ (Table 4 ). The site counseling explained $8 \%$ of the variance in intentions $(p<0.001)$, and attitudes explained $52 \%$ of the variance in intentions $(p<0.001 ;)$. Attitudes which predicted intentions include: MRO, self-efficacy, positive outcome expectancies, and an interaction between mattering and group. While controlling for site counseling and other attitudes, a one standard deviation change in monitoring role orientation, self-efficacy, and positive outcome expectancies would result respectively in a $0.30,0.28$, or 0.21 standard deviation change in intentions. The impact of mattering differed between the Diabetes Check groups (Figure 2). For students in the control group, as their sense of mattering increased, their intentions to ask about the diabetes ABCs were higher. For students in the intervention group, as their sense of mattering increased, their intentions were lower.

\section{Behavior Regression -Asking about the ABCs}

Hierarchical stepwise multiple linear regression was used to determine what items predicted students' behaviors in asking about the diabetes ABC. Two models were created to account for group differences in behaviors. The first model assessed predictors of A1c because the intervention group had greater improvements in asking about A1c (2). The second model assessed predictors of blood pressure and cholesterol combined as they did not have group differences (2).

Order of entry for the regression analysis reflected the conceptual model. Student situation variables were entered in variable block one, attitude variables in variable block two, and intention in variable block three. In addition, the mattering and group interaction term was included as a fourth variable block. The complete regression model accounted for a significant amount of variance in intentions, with $F(4,110)=20.8$, $\mathrm{p}<0.001, \mathrm{R}^{2}=0.43$ adjusted $\mathrm{R}^{2}=0.41$ (Table 5). 
Table 3 Correlation Matrix by Study Group

\begin{tabular}{|c|c|c|c|c|c|c|c|c|c|c|c|}
\hline \multicolumn{3}{|l|}{$\begin{array}{l}\text { Study } \\
\text { Group } \dagger\end{array}$} & 1 & 2 & 3 & 4 & 5 & 6 & 7 & 8 & 9 \\
\hline \multirow{2}{*}{$\begin{array}{l}\text { Ask about } \\
\text { A }\end{array}$} & \multirow{2}{*}{1} & $\mathrm{C}$ & $\begin{array}{l}1 \\
\mathrm{n}=56\end{array}$ & & & & & & & & \\
\hline & & DC & $\begin{array}{l}1 \\
\mathrm{n}=61\end{array}$ & & & & & & & & \\
\hline \multirow{2}{*}{$\begin{array}{l}\text { Ask about } \\
\text { B }\end{array}$} & \multirow{2}{*}{2} & $\mathrm{C}$ & $\begin{array}{l}0.70^{*} \\
\mathrm{n}=56\end{array}$ & $\begin{array}{l}1 \\
n=57\end{array}$ & & & & & & & \\
\hline & & DC & $\begin{array}{l}0.63^{*} \\
\mathrm{n}=61\end{array}$ & $\begin{array}{c}1 \\
\mathrm{n}=61\end{array}$ & & & & & & & \\
\hline \multirow{2}{*}{$\begin{array}{l}\text { Ask about } \\
\text { C }\end{array}$} & \multirow{2}{*}{3} & C & $\begin{array}{l}0.66^{*} \\
\mathrm{n}=57\end{array}$ & $\begin{array}{l}0.76^{*} \\
\mathrm{n}=57\end{array}$ & $\begin{array}{c}1 \\
n=57\end{array}$ & & & & & & \\
\hline & & DC & $\begin{array}{l}0.58^{*} \\
\mathrm{n}=61\end{array}$ & $\begin{array}{l}0.78^{*} \\
\mathrm{n}=61\end{array}$ & $\begin{array}{l}1 \\
n=61\end{array}$ & & & & & & \\
\hline \multirow{2}{*}{ Intention } & \multirow{2}{*}{4} & $\mathrm{C}$ & $\begin{array}{l}0.49 * \\
n=55\end{array}$ & $\begin{array}{l}0.52 * \\
\mathrm{n}=56\end{array}$ & $\begin{array}{l}0.40^{*} \\
\mathrm{n}=56\end{array}$ & $\begin{array}{c}1 \\
n=57\end{array}$ & & & & & \\
\hline & & DC & $\begin{array}{l}0.55^{*} \\
\mathrm{n}=61\end{array}$ & $\begin{array}{l}0.48^{*} \\
\mathrm{n}=61\end{array}$ & $\begin{array}{l}0.46^{*} \\
\mathrm{n}=61\end{array}$ & $\begin{array}{c}1 \\
\mathrm{n}=61\end{array}$ & & & & & \\
\hline \multirow{2}{*}{$\begin{array}{l}\text { Self- } \\
\text { Efficacy }\end{array}$} & \multirow{2}{*}{5} & $\mathrm{C}$ & $\begin{array}{l}0.68^{*} \\
\mathrm{n}=56\end{array}$ & $\begin{array}{l}0.72 * \\
\mathrm{n}=57\end{array}$ & $\begin{array}{l}0.62 * \\
n=57\end{array}$ & $\begin{array}{l}0.63^{*} \\
\mathrm{n}=57\end{array}$ & $\begin{array}{c}1 \\
n=58\end{array}$ & & & & \\
\hline & & DC & $\begin{array}{l}0.45^{*} \\
\mathrm{n}=61\end{array}$ & $\begin{array}{l}0.51^{*} \\
\mathrm{n}=61\end{array}$ & $\begin{array}{l}0.48^{*} \\
\mathrm{n}=61\end{array}$ & $\begin{array}{l}0.69 * \\
\mathrm{n}=61\end{array}$ & $\begin{array}{l}1 \\
n=61\end{array}$ & & & & \\
\hline \multirow{2}{*}{$\begin{array}{l}\text { Positive } \\
\mathrm{OE}^{* *}\end{array}$} & \multirow{2}{*}{6} & $\mathrm{C}$ & $\begin{array}{l}0.38 * \\
\mathrm{n}=55\end{array}$ & $\begin{array}{l}0.36 * \\
n=56\end{array}$ & $\begin{array}{l}\mathbf{0 . 2 2} \\
\mathrm{n}=56\end{array}$ & $\begin{array}{l}0.64 * \\
\mathrm{n}=57\end{array}$ & $\begin{array}{l}0.50^{*} \\
\mathrm{n}=57\end{array}$ & $\begin{array}{l}1 \\
\mathrm{n}=57\end{array}$ & & & \\
\hline & & DC & $\begin{array}{l}0.29 * \\
\mathrm{n}=61\end{array}$ & $\begin{array}{l}0.37 * \\
\mathrm{n}=61\end{array}$ & $\begin{array}{l}\mathbf{0 . 2 5} \\
\mathrm{n}=61\end{array}$ & $\begin{array}{l}0.48^{*} \\
\mathrm{n}=61\end{array}$ & $\begin{array}{l}0.43^{*} \\
\mathrm{n}=61\end{array}$ & $\begin{array}{l}1 \\
\mathrm{n}=61\end{array}$ & & & \\
\hline \multirow{2}{*}{$\begin{array}{l}\text { Negative } \\
\mathrm{OE}^{* *}\end{array}$} & \multirow{2}{*}{7} & $\mathrm{C}$ & $\begin{array}{l}-0.35 * \\
n=55\end{array}$ & $\begin{array}{l}-0.25^{*} \\
\mathrm{n}=56\end{array}$ & $\begin{array}{l}-0.27 * \\
\mathrm{n}=56\end{array}$ & $\begin{array}{l}-0.39 \\
n=57\end{array}$ & $\begin{array}{l}-0.47 * \\
\mathrm{n}=57\end{array}$ & $\begin{array}{l}-\mathbf{0 . 2 4} \\
n=57\end{array}$ & $\begin{array}{l}1 \\
n=57\end{array}$ & & \\
\hline & & DC & $\begin{array}{l}-0.42 * \\
n=61\end{array}$ & $\begin{array}{l}-0.42^{*} \\
\mathrm{n}=61\end{array}$ & $\begin{array}{l}-0.42 * \\
n=61\end{array}$ & $\begin{array}{l}-0.45 \\
n=61\end{array}$ & $\begin{array}{l}-0.56^{*} \\
n=61\end{array}$ & $\begin{array}{l}-0.49 * \\
n=61\end{array}$ & $\begin{array}{l}1 \\
n=61\end{array}$ & & \\
\hline \multirow{2}{*}{ CRO } & \multirow{2}{*}{8} & $\mathrm{C}$ & $\begin{array}{l}\mathbf{0 . 1 5} \\
n=55\end{array}$ & $\begin{array}{l}\mathbf{0 . 2 6} \\
\mathrm{n}=56\end{array}$ & $\begin{array}{l}\mathbf{0 . 0 8} \\
n=56\end{array}$ & $\begin{array}{l}0.52 * \\
\mathrm{n}=57\end{array}$ & $\begin{array}{l}0.29 * \\
n=57\end{array}$ & $\begin{array}{l}0.37 * \\
\mathrm{n}=57\end{array}$ & $\begin{array}{l}-0.49 * \\
n=57\end{array}$ & $\begin{array}{l}1 \\
n=57\end{array}$ & \\
\hline & & DC & $\begin{array}{l}\mathbf{- 0 . 0 1} \\
\mathrm{n}=61\end{array}$ & $\begin{array}{l}\mathbf{- 0 . 0 5} \\
\mathrm{n}=61\end{array}$ & $\begin{array}{l}\mathbf{- 0 . 0 6} \\
n=61\end{array}$ & $\begin{array}{l}\mathbf{0 . 1 3} \\
\mathrm{n}=61\end{array}$ & $\begin{array}{l}\mathbf{0 . 1 2} \\
\mathrm{n}=61\end{array}$ & $\begin{array}{l}\mathbf{0 . 0 7} \\
\mathrm{n}=61\end{array}$ & $\begin{array}{l}\mathbf{- 0 . 1 7} \\
\mathrm{n}=61\end{array}$ & $\begin{array}{l}1 \\
\mathrm{n}=61\end{array}$ & \\
\hline \multirow{2}{*}{ MRO } & \multirow{2}{*}{9} & $\mathrm{C}$ & $\begin{array}{l}0.55^{*} \\
\mathrm{n}=55\end{array}$ & $\begin{array}{l}0.60^{*} \\
\mathrm{n}=56\end{array}$ & $\begin{array}{l}0.53^{*} \\
\mathrm{n}=56\end{array}$ & $\begin{array}{l}0.66^{*} \\
\mathrm{n}=57\end{array}$ & $\begin{array}{l}0.77^{*} \\
\mathrm{n}=57\end{array}$ & $\begin{array}{l}0.57^{*} \\
\mathrm{n}=57\end{array}$ & $\begin{array}{l}-0.44^{*} \\
\mathrm{n}=57\end{array}$ & $\begin{array}{l}0.42^{*} \\
\mathrm{n}=57\end{array}$ & $\begin{array}{l}1 \\
\mathrm{n}=57\end{array}$ \\
\hline & & DC & $\begin{array}{l}0.56 * \\
n=61\end{array}$ & $\begin{array}{l}0.56^{*} \\
\mathrm{n}=61\end{array}$ & $\begin{array}{l}0.55^{*} \\
\mathrm{n}=61\end{array}$ & $\begin{array}{l}0.67 * \\
\mathrm{n}=61\end{array}$ & $\begin{array}{l}0.70^{*} \\
\mathrm{n}=61\end{array}$ & $\begin{array}{l}0.53 * \\
n=61\end{array}$ & $\begin{array}{l}-0.58^{*} \\
\mathrm{n}=61\end{array}$ & $\begin{array}{l}\mathbf{0 . 1 5} \\
\mathrm{n}=61\end{array}$ & $\begin{array}{l}1 \\
n=61\end{array}$ \\
\hline \multirow{2}{*}{ Mattering } & \multirow{2}{*}{10} & $\mathrm{C}$ & $\begin{array}{l}0.45^{*} \\
\mathrm{n}=56\end{array}$ & $\begin{array}{l}0.58^{*} \\
\mathrm{n}=57\end{array}$ & $\begin{array}{l}0.35^{*} \\
n=57\end{array}$ & $\begin{array}{l}0.69 * \\
n=57\end{array}$ & $\begin{array}{l}0.63^{*} \\
\mathrm{n}=58\end{array}$ & $\begin{array}{l}0.59 * \\
n=57\end{array}$ & $\begin{array}{l}-0.37 * \\
n=57\end{array}$ & $\begin{array}{l}0.60^{*} \\
\mathrm{n}=57\end{array}$ & $\begin{array}{l}0.54 * \\
\mathrm{n}=57\end{array}$ \\
\hline & & DC & $\begin{array}{l}\mathbf{- 0 . 0 2} \\
n=61\end{array}$ & $\begin{array}{l}\mathbf{0 . 0 7} \\
\mathrm{n}=61\end{array}$ & $\begin{array}{l}\mathbf{0 . 0 0} \\
n=61\end{array}$ & $\begin{array}{l}\mathbf{- 0 . 1 7} \\
n=61\end{array}$ & $\begin{array}{l}\mathbf{0 . 0 9} \\
\mathrm{n}=61\end{array}$ & $\begin{array}{l}\mathbf{0 . 1 6} \\
\mathrm{n}=61\end{array}$ & $\begin{array}{l}\mathbf{- 0 . 1 9} \\
n=61\end{array}$ & $\begin{array}{l}\mathbf{0 . 0 7} \\
n=61\end{array}$ & $\begin{array}{l}\mathbf{0 . 0 8} \\
n=61\end{array}$ \\
\hline
\end{tabular}

(Non-significant correlations are bolded ${ }^{*}$ Correlation is significant $(\mathrm{p}<0.05) .{ }^{*} \mathrm{OE}=$ Outcome Expectancies †Diabetes Check Assignment: DC=Diabetes Check Assignment and C=Control; for DC $n=61$ for all cells. For $\mathrm{c} n=$

When controlling for group, each one standard deviation change in site counseling increased asking about A1c by 0.38 standard deviations. When controlling for site counseling, students in the intervention group had a 0.58 higher score in behavior asking about A1c on a scale from one to seven. Together, site counseling and group explained $21 \%$ of the variance in behavior asking about A1c $(\mathrm{p}<0.001)$. Attitudes (i.e., self-efficacy and MRO) explained 23\% of the variance in asking about A1c $(p<0.001)$. For each one standard deviation change in self-efficacy or MRO, asking about A1c increased by 0.33 or 0.22 standard deviations. 
Table 4 Model Coefficients Predicting Intentions

\begin{tabular}{|l|l|c|c|c|c|}
\hline \multirow{2}{*}{ Block } & \multirow{2}{*}{ Independent Variable } & \multicolumn{2}{|c|}{$\begin{array}{c}\text { Unstandardized } \\
\text { Coefficients }\end{array}$} & $\begin{array}{c}\text { Standardized } \\
\text { Coefficients Beta }\end{array}$ & \multirow{2}{*}{ Semi-Partial r } \\
\cline { 3 - 5 } & B & Std. Error & \\
\hline One & Site Counseling & 0.146 & 0.051 & $0.256^{*}$ & 0.256 \\
\hline & $\begin{array}{l}\text { Diabetes Check } \\
\text { Assignment }\end{array}$ & 0.152 & 0.134 & 0.102 & 0.102 \\
\hline Two & Mattering x group & -0.683 & 0.186 & $-0.294^{*}$ & -0.221 \\
\hline & MRO & 0.238 & 0.078 & $0.295^{*}$ & 0.185 \\
\hline & Mattering & 0.277 & 0.130 & $0.196^{*}$ & 0.129 \\
\hline & Self-efficacy & 0.177 & 0.060 & $0.277^{*}$ & 0.178 \\
\hline & Positive OE** & 0.187 & 0.067 & $0.214^{*}$ & 0.167 \\
\hline
\end{tabular}

The model used $n=117$. The following variables were not significant: block-ease of use and block two -CRO and negative outcome expectancies. Data were checked and met assumptions for linearity, independence, normality of error, and heteroscedasticity.

$* \mathrm{p}<0.05$.

** $\mathrm{OE}=$ Outcome Expectancies.

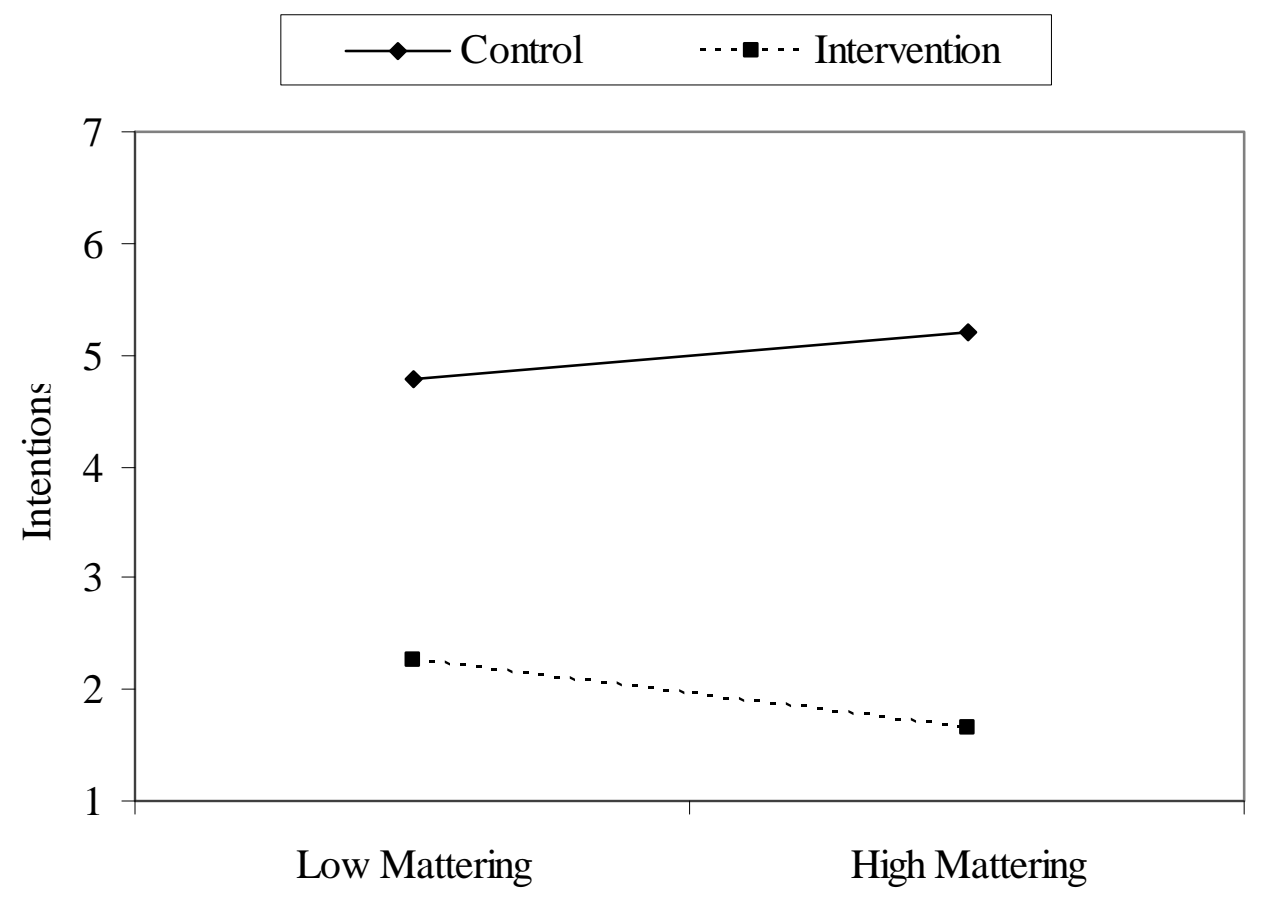

Figure 2. Mattering and Group Interactions I 
Table 5 Model Coefficients Predicting Asking about A1c

\begin{tabular}{|l|l|l|l|l|l|}
\hline \multirow{2}{*}{$\begin{array}{l}\text { Variable } \\
\text { Block }\end{array}$} & $\begin{array}{l}\text { Independent } \\
\text { Variable }\end{array}$ & $\begin{array}{l}\text { Unstandardized } \\
\text { Coefficients }\end{array}$ & $\begin{array}{l}\text { Standardized } \\
\text { Coefficients Beta }\end{array}$ & Semi-Partial r \\
\hline One & B Std. Error & & 0.380 \\
\hline & Site Counseling & 0.370 & 0.082 & $0.380^{*}$ & 0.226 \\
\hline & $\begin{array}{l}\text { Diabetes Check } \\
\text { Assignment }\end{array}$ & 0.579 & 0.215 & $0.227^{*}$ & 0.223 \\
\hline Two & Self-Efficacy & 0.360 & 0.116 & $0.330^{*}$ & 0.148 \\
\hline
\end{tabular}

The model used $n=115$. The following variables were not significant: block one-gender and ease of use, block two-CRO and mattering, and block 3- intentions. Data were checked and met assumptions for linearity, independence, normality of error, and heteroscedasticity.

${ }^{*} \mathrm{p}<0.05$.

Table 6 Model Coefficients Predicting Asking about Blood Pressure \& Cholesterol

\begin{tabular}{|l|l|l|l|l|l|}
\hline \multirow{2}{*}{$\begin{array}{l}\text { Variable } \\
\text { Block }\end{array}$} & $\begin{array}{l}\text { Independent } \\
\text { Variable }\end{array}$ & \multicolumn{2}{|l|}{ Unstandardized Coefficients } & $\begin{array}{l}\text { Standardized } \\
\text { Coefficients Beta }\end{array}$ & Semi-Partial r \\
\cline { 2 - 5 } & B & Std. Error & \\
\hline One & Site Counseling & 0.340 & 0.079 & $0.374^{*}$ & 0.374 \\
\hline Two & Self-Efficacy & 0.384 & 0.106 & $0.376^{*}$ & 0.255 \\
\hline & MRO & 0.345 & 0.137 & $0.265^{*}$ & 0.178 \\
\hline
\end{tabular}

The model used $\mathrm{n}=116$. The following variables were not significant: block one-diabetes check assignment, gender and ease of use, block two-CRO and mattering, and block 3- intentions. Data were checked and met assumptions for linearity, independence, normality of error, and heteroscedasticity.

${ }^{*} \mathrm{p}<0.05$.

For blood pressure and cholesterol combined, the final regression model accounted for a significant amount of variance in behavior in asking about blood pressure and cholesterol, with $\mathrm{F}(3,112)=29.6$, $\mathrm{p}<0.001, \mathrm{R}^{2}=0.44$ adjusted $\mathrm{R}^{2}=0.43$ (Table 6). Pharmacy site counseling explained $13 \%$ of the variance in behavior asking about blood pressure and cholesterol $(\mathrm{p}<0.001)$. Student attitudes explained a further $30 \%(\mathrm{p}<0.001)$. A one standard deviation change in the site counseling variable increased asking about blood pressure and cholesterol by 0.40 standard deviations. Similarly, when controlling for site, one standard deviation change in either self-efficacy or MRO increased asking about blood pressure and cholesterol by 0.38 or 0.27 standard deviations, respectively.

\section{DISCUSSION}

In summary, pharmacy students' intentions and monitoring behaviors were predicted by the students' situation and attitudes. For research question one, students' intentions to ask patients about the diabetes ABCs were predicted by pharmacy site counseling, monitoring role beliefs, self-efficacy, and positive outcome expectancies. Mattering predicted intentions, but differently in each study group. For research question two, behavior in asking about the Diabetes ABCs was predicted by pharmacy site counseling, self-efficacy, and monitoring role beliefs. Students' behavior in asking about A1c was also predicted by completing the Diabetes Check assignment. 
The pharmacy site counseling, self-efficacy and monitoring role beliefs orientation were consistent predictors of intentions and behavior. Pharmacy site counseling variable consisted of two questions on expectations for counseling and preceptor's frequency of asking about the diabetes ABCs.

Self-efficacy is the cornerstone of Bandura's Social Cognitive Theory and is the most widely employed construct in this theory. In a multitude of differing settings, self-efficacy has been shown to be a strong predictor of behavior ( 7 , 17, 18). This research reaffirms that finding with senior students in a community pharmacy setting. A students' self-efficacy toward asking about the Diabetes ABCs under a variety of conditions was a strong predictor of both intentions and behaviors. Self-efficacy has been found to predict pharmaceutical care behavior, though this relationship was moderated by behavioral control (19-21). This supports the continued use of selfefficacy to evaluate pharmacy curriculum (8).

This research supports the growing body of literature demonstrating how pharmacists' role beliefs influence patient-pharmacist interactions (22-29). This research supports that body of literature by using amore specific measure, MRO, to assess that relationship between a more specific behavior, monitoring and behavior.

In addition to self-efficacy and MRO, there were several other predictors of behavior and intentions. Intentions to ask about the diabetes $\mathrm{ABCs}$ in the future were also predicted by positive outcome expectancies and a mattering and Diabetes Check Assignment interaction term. Independent of self-efficacy, outcome expectancies predicted intention to perform a behavior in the future. Thus, when assessing which pharmacists intend to perform a given monitoring activity, it may be important to assess their expectations for outcomes. Mattering was positively related with intentions to monitor for students who did not monitor the diabetes ABCs in the Diabetes Check Assignment. Similarly, pharmacists with higher levels of mattering where found to desire spending a greater proportion of time in consultation (30). However, intentions to monitor were not related to mattering for students who completed the Diabetes Check Assignment. Mattering, arises out of the immediate interaction. Experiences with the Diabetes Check assignment may have refocused students away from this immediate benefit of the Diabetes Check and instead students considered the other benefits of monitoring. Other potential reasons fort this interaction include an unmeasured interaction between mattering and another variable or measurement error.

It is encouraging that role beliefs may be stronger predictors of who will ask about diabetes ABCs in the future than a students' sense of mattering to a patient. Professionals such as pharmacists should perform activities that will help their patients, and sometimes that entails helping people learn what they need to do. Patients' needs and concerns should take precedence, but additional care (i.e., monitoring) should not be withheld because students were driven by a sense of mattering rather than professional duty to their patients.

\section{Conceptual Model}

The conceptual model was adapted in light of findings. First, the conceptual model was spilt into two models. One predicts intentions to perform the behavior in the future (Figure 3) and the other predicts current behaviour (Figure 4).

In past research, intentions have been positively related to pharmacists' pharmaceutical care behaviors (31). However, students' intentions for future behavior may be less likely to be related to their current clerkship behavior, as students may not have decided their future career path. Gender and ease of use were dropped from the conceptual models, as they did not predict either intentions or behaviors. Pharmacy site counseling, self-efficacy and monitoring role beliefs were retained in both models due to their consistent relationships. Positive outcome expectancies were retained in the model to predict intentions, though their inclusion in future research should be weighed against the response burden. Mattering was a predictor of intentions, although it was a positive predictor for the control group and did not predict intentions in the intervention group.

These models may not capture all predictors of monitoring intentions and behaviors. First, future changes in behavior may affect other changes in attitudes that can once more alter behavior. 
Figure 3 Conceptual Model: Predictors of Students' Future Intentions to Ask about the Diabetes ABCs in the Future

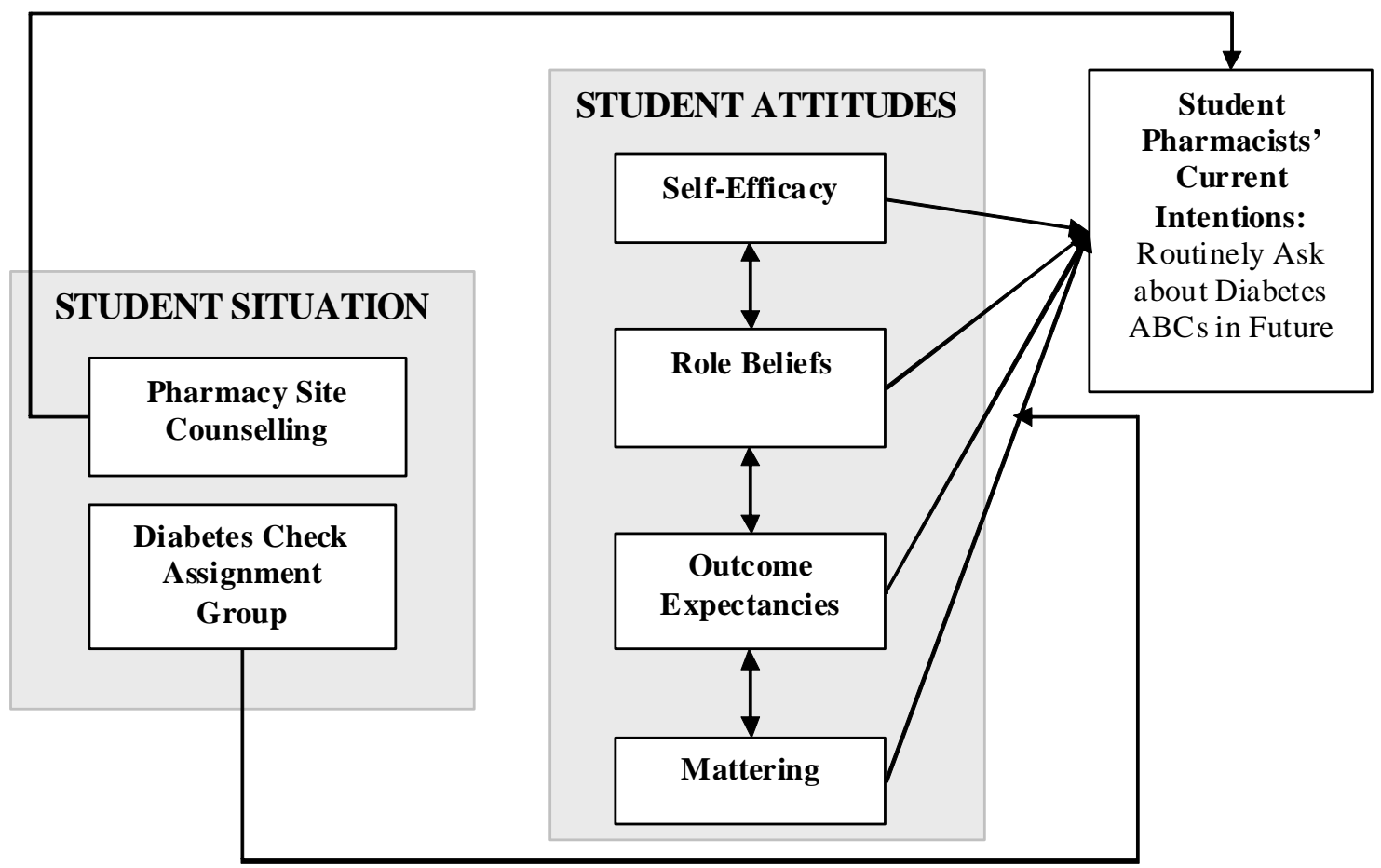

Figure 4 Conceptual Model: Predictors of Students’ Current Behavior

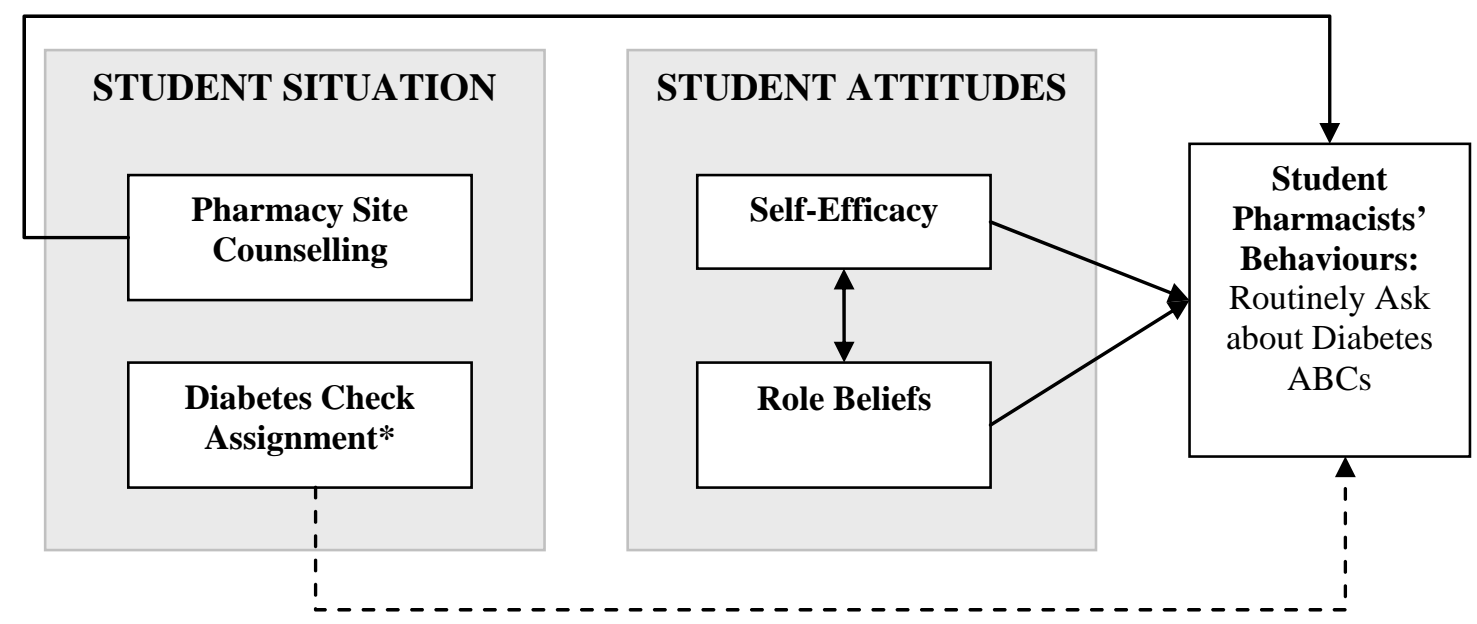

* Intervention effect is present when behavior is low at baseline, such as asking about A1c, not blood pressure or cholesterol.

Anecdotal reports from pharmacy educators and clinical instructors have suggested that, in some cases, there is uptake of these monitoring behaviors in pharmacy sites. The feedback of this uptake on students' subsequent behavior may not be captured. While these models assessed the influence of pharmacy site counseling behaviors, a more systematic examination of organizational factors 
such as pharmacy policies, relationships with other health care providers, technicians' roles, and pharmacy reimbursement should be considered if a more comprehensive model of pharmacist behavior is to be considered (32).

\section{CONCLUSIONS}

Monitoring intentions and behaviors were influenced by pharmacy site counseling, monitoring role beliefs, and self-efficacy and future research investigating the pharmacy students' behavior should include these variables. Future research may consider assessing if these factors are also robust predictors of pharmacists' monitoring behaviors. The role of mattering and outcome expectancies in predicting monitoring intentions requires further study.

\section{REFERENCES}

[1]. AACP . White paper on pharmacy student professionalism. American Pharmaceutical Association Academy of Students of pharmacy-American Association of Colleges of Pharmacy council of deans task force on professionalism. $\mathrm{J}$ Am Pharm Assoc, 2000 Jan-Feb;40(1):96-102.

[2]. Guirguis LM, Chewning BA, Kieser MA. A randomized controlled trial to evaluate the impact of structured patient interactions on pharmacy students' counseling beliefs and behaviors. Int J Pharm Pract. 2009;17:53-60.

[3]. NDEP's publication catalog [homepage on the Internet]. . 2005. Available from: http://www.ndep.nih.gov/diabetes/pubs/catalog. htm\#PubsHCCont.

[4]. WHO | diabetes [homepage on the Internet]. [cited 7/17/2008]. Available from: http://www.who.int/diabetes/en/.

[5]. Zarembski DG, Boyer JG, Vlasses PH. A survey of advanced community pharmacy practice experiences in the final year of the PharmD curriculum at US colleges and schools of pharmacy. Am $\mathrm{J}$ Pharm Educ. 2005;69(1):Article: 02.

[6]. Siracuse MV, Schondelmeyer SW, Hadsall RS, Schommer JC. Survey of pharmacy student work experience. $\mathrm{J} \quad \mathrm{Am}$ PharmAssoc,2004;44(2):293.

[7]. Bandura A. Self-efficacy: The exercise of control. New York: W.H. Freeman; 1997.

[8]. Plaza CM, Draugalis JR, Retterer J, Herrier RN. Curricular evaluation using self-efficacy measurements. Am J Pharm Educ. 2002;66(1):51-54.

[9]. Wongwiwatthananukit S, Newton GD, Popovich NG. Development and validation of an instrument to assess the self-confidence of students enrolled in the advanced pharmacy practice experience. Am J Pharm Educ. 2002;66(1):5-19.

[10]. Guirguis LM, Chewning BA. Role theory: Literature review and implications for patientpharmacist interactions. Res Soc Admin Pharm. 2005 12;1(4):483-507.

[11]. Connolly KM, Myers JE. Wellness and mattering: The role of holistic factors in job satisfaction. J Employ Counsel. 2003;40:152160.

[12]. Marcus FM, Rosenberg M. In: Mattering: Its measurement and significance in everyday life. Eastern sociological society meetings; 1987.

[13]. Guirguis LM, Chewning BA. Development of a measure to assess pharmacy students' beliefs about monitoring chronic diseases. Res Social Adm Pharm,2008 12/01;4(4):402-16.

[14]. Resnick B. The impact of self-efficacy and outcome expectations on functional status in older adults. Top Geriatr Rehabil. 2002;17(4):110.

[15]. Resnick B, Zimmerman SI, Orwig D, Furstenberg AL, Magaziner J. Outcome expectations for exercise scale: Utility and psychometrics. J Gerontol B Psychol Sci Soc Sci. 2000 Nov;55(6):S352-6.

[16]. Resnick B. Efficacy beliefs in geriatric rehabilitation. J Gerontol Nurs. 1998 Jul; 24(7):34-44.

[17]. Bandura A. Social foundations of thought and action: A social cognitive theory. Englewood Cliffs, N.J.: Prentice-Hall; 1986.

[18]. Bandura A. Self-efficacy. In: V.S. R, editor. Encyclopedia of human behavior. New York: Academic Press; 1998. p. 71-81.

[19]. Hudmon KS, Prokhorov AV, Corelli RL. Tobacco cessation counseling: Pharmacists' opinions and practices. P t Ed Counsel. 2006 61(1):152-60.

[20]. Martin BA. Developing and evaluating pharmacists' confidence and skills in a continuing education program [dissertation]. Madison, Wisconsin: University of WisconsinMadison; 2003.

[21]. Farris KB, Schopflocher DP. Between intention and behavior: An application of community pharmacists' assessment of pharmaceutical care. Soc Sci Med. 1999 Jul;49(1):55-66.

[22]. Pendergast JF, Kimberlin CL, Berardo DH, McKenzie LC. Role orientation and community 
pharmacists' participation in a project to improve patient care. Soc Sci Med. 1995 Feb;40(4):557-65.

[23]. Desselle S. New york state pharmacists: Relationship between job satisfaction, workplace climate, commitment, and providing pharmaceutical care. N Y State Pharm Century II. 1998;72(4):24, 27-29.

[24]. Schommer JC, Wiederholt JB. Pharmacists' perceptions of patients' needs for counseling. Am J Hosp Pharm. 1994;51(4):478-85.

[25]. Schommer JC, Wiederholt JB. A field investigation of participant and environment effects on pharmacist-patient communication in community pharmacies. Med Care. 1995 Jun;33(6):567-84.

[26]. Schommer JC. Effects of interrole congruence on pharmacist-patient behavior. Health Commun. 1994;6:297-309.

[27]. Schommer JC, Wiederholt JB. The association of prescription status, patient age, patient gender, and patient question asking behavior with the content of pharmacist-patient communication. Pharm Res. 1997;14(2):145-51.

[28]. Mason HL, Svarstad BL. Medication counseling behaviors and attitudes of rural community pharmacists. Drug Intell Clin Pharm. 1984;18(5):409-14.

[29]. Mason HL. The pharmacist's medication counseling role: Attitudes and behavior of community practitioners [dissertation]. University of Wisconsin-Madison; 1979.

[30]. Guirguis LM, Kreling DH. Wisconsin pharmacists believe they matter to their patients? Journal of the Pharmacy Society of Wisconsin. 2007:44-48.

[31]. Odedina FT, Segal R. Behavioral pharmaceutical care scale for measuring pharmacists' activities. Am J Health-Syst Pharm. 1996;53:855-865.

[32]. Roberts AS, Benrimoj SI, Chen TF, Williams KA, Aslani P. Implementing cognitive services in community pharmacy: A review of models and frameworks for change. Int J Pharm Pract. 2006;14:105-13. 